\title{
Retiro de las precauciones específicas de aislamiento de los pacientes con infección por SARS-CoV-2 en entornos hospitalarios
}

\author{
Withdrawal of specific isolation precautions for patients \\ with SARS-CoV-2 infection in hospital settings \\ Eduardo Arias de la Garza,* Hilda Hernández Orozco* \\ * Departamento de Infectología Pediátrica y Comité de Infecciones Asociadas a la Atención en Salud INP.
}

La información disponible en la literatura indica que los pacientes con COVID-19 leve a moderado ya no son infectantes posterior a 10 días del inicio de los síntomas. La mayoría de los pacientes con enfermedad más grave o en estado crítico, además de los inmunocomprometidos, probablemente siguen siendo infectantes por no más de 20 días después de la aparición de los síntomas; sin embargo, han habido varios informes de pacientes que excretan virus con capacidad de replicación más allá de los 20 días debido al estado de inmunodepresión grave.

Los pacientes recuperados pueden continuar arrojando ARN del SARS-CoV-2 detectable, pero no infeccioso, en muestras de las vías respiratorias superiores hasta tres meses después del inicio de la enfermedad, aunque en concentraciones considerablemente más bajas que durante la enfermedad y en rangos de concentración en los que no se ha detectado el virus competente para la replicación. ${ }^{2-5}$

Las circunstancias que dan como resultado un ARN del SARS-CoV-2 detectable de forma persistente aún no se han determinado. Los estudios no han encontrado evidencia de que los pacientes clínicamente recuperados con persistencia del
ARN viral hayan transmitido el SARS-CoV-2 a otras personas. Estos hallazgos refuerzan la justificación para confiar en una estrategia basada en síntomas, más que basada en pruebas, para terminar con el aislamiento de la mayoría de los pacientes, de modo que no se mantengan innecesariamente aislados y excluidos del trabajo u otras actividades. . $^{6} 8$

Las decisiones sobre la suspensión de las precauciones específicas de aislamiento para pacientes con infección por SARS-CoV-2 deben tomarse en el contexto de las circunstancias locales. En general, se debe utilizar una estrategia basada en síntomas, ya no se recomienda una estrategia basada en pruebas porque, en la mayoría de los casos, da como resultado un aislamiento prolongado de los pacientes que continúan arrojando partículas de ARN del SARSCoV-2 detectable, pero que ya no es infectante. Asimismo, es importante siempre valorar que el periodo de tiempo utilizado depende de la gravedad de la enfermedad del paciente y de su estado de inmunocompromiso. ${ }^{9-11}$

Recomendaciones: (Centers for Disease Control and Prevention)

Citar como: Arias de la Garza E, Hernández OH. Retiro de las precauciones específicas de aislamiento de los pacientes con infección por SARS-CoV-2 en entornos hospitalarios. Rev Latin Infect Pediatr. 2021; 34 (3): 115-117. https://dx.doi.org/10.35366/102232

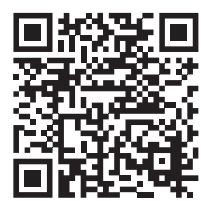


1. Duración de las precauciones específicas de aislamiento

- Para la mayoría de los adultos con enfermedad por COVID-19 el aislamiento y las precauciones se pueden suspender 10 días después del inicio de los síntomas y después de la resolución de la fiebre durante al menos 24 horas, sin el uso de medicamentos para reducir la misma y con la mejoría de otros síntomas (tos, dificultad para respirar).

- Algunos pacientes con enfermedad grave pueden producir virus con capacidad de replicación más allá de los 10 días, lo que puede justificar la prolongación de la duración del aislamiento y las precauciones hasta 20 días después del inicio de los síntomas; los pacientes gravemente inmunodeprimidos (oncológicos, trasplantados, VIH, uso de medicamentos inmunosupresores) pueden producir virus con capacidad de replicación más allá de los 20 días y requieren pruebas adicionales y valoración con especialistas en enfermedades infecciosas y expertos en control de infecciones.

- Para los pacientes que nunca desarrollan síntomas, las precauciones específicas de aislamiento pueden suspenderse 10 días después de la fecha del primer resultado positivo de la prueba RT-PCR para ARN del SARS-CoV-2.

2. Pruebas de diagnóstico viral (RT-PCR o antígeno) para interrumpir el aislamiento o las precauciones

- Para los pacientes que están gravemente inmunodeprimidos se podría considerar una estrategia basada en pruebas en consulta con expertos en enfermedades infecciosas.

- Para todos los demás ya no se recomienda una estrategia basada en pruebas, excepto para interrumpir el aislamiento antes de lo que ocurriría con la estrategia descrita en el punto número uno.

\section{Pruebas serológicas}

- Aunque las pruebas serológicas que indican la presencia de anticuerpos contra el SARS-CoV-2 pueden significar una infección previa o en resolución, generalmente no deben usarse para establecer la presencia o ausencia de una infección aguda por el SARS-CoV-2.

- Por lo general, la fecha de una prueba serológica positiva no debe usarse para determinar el inicio del periodo de 90 días después de la infección por SARS-CoV-2 para la cual no se recomienda repetir la prueba o poner en cuarentena al paciente.

La implementación de estas recomendaciones junto con las estrategias de prevención actuales para la transmisión del SARS-CoV-2 (uso de cubrebocas, distanciamiento social, evitar las multitudes y realizar higiene de manos en los cinco momentos) y los riesgos de la posible infección por SARS-CoV-2 de pacientes recuperados es, por lo general, demasiado baja para justificar la repetición de la prueba y el aislamiento preventivo.

\section{REFERENCIAS}

1. Arons MM, Hatfield KM, Reddy SC, Kimball A, James A, Jacobs JR et al. Presymptomatic SARSCoV-2 infections and transmission in a skilled nursing facility. $\mathrm{N}$ Engl $\mathrm{J}$ Med. 2020; 382 (22): 2081-2090.

2. Bullard J, Durst K, Funk D, Strong JE, Alexander D, Garnett $\mathrm{L}$ et al. Predicting Infectious SARSCoV-2 from diagnostic samples. Clin Infect Dis. 2020; 71 (10): 2663-2666.

3. Cheng HW, Jian SW, Liu DP, Ng TC, Huang WT, Lin HH et al. Contact tracing assessment of COVID-19 transmission dynamics in Taiwan and risk at different exposure periods before and after symptom onset. JAMA Intern Med. 2020; 180 (9): 1156-1163.

4. Kiyuka PK, Agoti CN, Munywoki PK, Njeru R, Bett A, Otieno $\mathrm{JR}$, et al. Human coronavirus NL63 molecular epidemiology and evolutionary patterns in rural 5 . Coastal Kenya. J Infect Dis. 2018; 217 (11): 1728-1739.

5. Corea Centers for Disease Control and Prevention. Findings from Investigation and Analysis of repositive cases. 2020.

6. Li N, Wang X, Lv T. Prolonged SARS-CoV-2 RNA Shedding: not a rare phenomenon. J Med Virol. 2020; 92 (11): 22862287.

7. Liu WD, Chang SY, Wang JT, Tsai MJ, Hung CC, Hsu CL et al. Prolonged virus shedding even after seroconversion in a patient with COVID-19. J Infect. 2020; 81 (2): 318-356.

8. Lu J, Peng J, Xiong Q, Liu Z, Lin H, Tan X et al. Clinical, immunological and virological characterization of COVID-19 patients that test re-positive for SARS-CoV-2 by RT-PCR. (Preprint) Medrxiv. 2020; 59: 102960.

9. Midgley CM, Kujawski SA, Wong KK, Collins, JP, Epstein L, Killerby ME et al. (2020). Clinical and virologic characteristics of the first 12 patients with coronavirus disease 2019 (COVID-19) in the United States. Nat Med. 2020; 26 (6): 861-868.

10. Quicke K, Gallichote E, Sexton N, Young M, Janich A, Gahm $G$ et al. Longitudinal surveillance for SARS-CoV-2 RNA 
Rev Latin Infect Pediatr. 2021; 34 (3): 115-117

among asymptomatic staff in five colorado skilled nursing facilities: epidemiologic, virologic and sequence analysis. (Preprint) Medrxiv. 2020; 2020.06.08.20125989.

11. Van Kampen J, Van de Vijver D, Fraaij P, Haagmans B, Lamers M, Okba N et al. Shedding of infectious virus in hospitalized patients with coronavirus disease-2019 (COVID-19): duration and key determinants. (Preprint) Medrxiv. 2020: 1-28.
Financiamiento: Ninguno.

Conflicto de intereses: Ninguno.

Correspondencia:

Eduardo Arias de la Garza

E-mail: Ialo_arias@hotmail.com 